\title{
Transmission Power and Effects on Energy Consumption and Performance in MANET
}

\author{
S. Blakeway ${ }^{1 *}$, A. Kirpichnikova ${ }^{2}$, M. Schaeffer ${ }^{3}$, E. L. Secco ${ }^{4}$ \\ ${ }^{1}$ Faculty of Science and Engineering, School of Computer Science, University of Manchester, UK \\ ${ }^{2}$ Department of Computing Science and Mathematics, University of Stirling, UK \\ ${ }^{3}$ Institut National Des Sciences Appliquees, INSA, France \\ ${ }^{4}$ Robotic Laboratory, Department of Mathematics \& Computer Science, Liverpool Hope University, Hope Park L16 9JD, UK
}

\begin{abstract}
This paper explores the effect that transmission power has on the performance of a Mobile Ad hoc Network (MANET). The goal of this research is to determine if the lifetime of the network can be prolongated by using less energy and thus, resulting in a more energy efficient 'greener' architecture. A total of 72 unique simulations are conducted of various configurations covering a large variety of possible scenarios: we examined configurations with a different number of nodes, number of traffic flows, mobility model, transmission power and geographical areas. Results show that there is an optimal transmission power, which enhances greater network performance: moreover, this optimal transmission setting makes the network more energy efficient in terms of depletion of the finite energy sources of the nodes. Our overall findings also confirm that higher transmission power results in less energy consumption
\end{abstract}

Keywords: MANET, Green Networks, Transmission Power Optimization, Energy Aware Networks.

Received on 15 October 2018, accepted on 06 December 2018, published on 31 January 2019

Copyright (C) 2019 S. Blakeway et al., licensed to EAI. This is an open access article distributed under the terms of the Creative Commons Attribution licence (http://creativecommons.org/licenses/by/3.0/), which permits unlimited use, distribution and reproduction in any medium so long as the original work is properly cited.

doi: 10.4108/eai.13-7-2018.159336

*Corresponding author. Email: Stewart.blakeway@manchester.ac.uk

\section{Introduction}

There has been much research of TPCPs (Transmission Power Control Protocols) in recent years, for example in an empirical study was conducted to determine a target TP (Transmission Power) based on an optimum TP [1]. The optimum TP was obtained using three link control properties and empirical data. In our research, we also consider one of the link control properties discussed which is that of Packet Delivery. We also consider the other link properties (Channel Quality and Channel Stability) but we do so transitively, i.e. based on the packet delivery ratio, if packets are being delivered it can be assumed that both Channel Quality and Channel Stability are sufficiently high for the successful transmission of data packets.

Authors in [2] give explanations to quantify the energy dissipated by the transmitting and receiving antennas and they describe the Heinselman-Chandrakasan-Balakrishnan (HCB) energy model [3]. Moreover, the authors of paper [2] give metrics of the power drain properties of state-of- the art radio antenna design. In our research, a Wi-fi Radio Energy Model was used that defines 4 states $T_{X}, R_{X}, I D L E$ and SLEEP. The default state being IDLE. The different types of transactions that are defined are detailed in [4]. The default values for power consumption are based on measurements reported in [5] and are discussed in more detail in the methodology.

There has been research conducted that detail the issues of selecting the transmission power in order to minimize the transmission power and thus conserve energy [6]. The work calculates the transmission power required to transmit n number of bits (which are converted to energy). The authors explain how the automatic repeat request (ARQ) protocol was used to establish link quality. The work also incorporates error percentage of the expected energy levels.

A similar approach can be observed in [7], where the authors have analysed the effect of transmission power with that of performance. The key differences are that their work discusses Internet of Things to produce large scale interconnection as justification for reducing energy where we are concerned with prolonging the lifetime of the 
network. Our work also differs because we are using a MANET whereby their work considered static sensor networks (two topologies). Unfortunately, it was unclear which simulation software was used and the other configuration parameters for their experiments. However, their results are promising and show that there is not a significant trade-off between performance and scaling up the transmission power. Also, mentioned in the future work section was that one may consider other routing protocols such as Ad hoc On-Demand Distance Vector (AODV), our research uses this routing protocol as well as the Destination-Sequenced Distance-Vector (DSDV).

Other related work has been conducted in [8] whereby authors considered energy consumption in sensor networks like that of the work of [7]. The authors explain that transmitting at a constant highest transmission power leads to energy wastage and causes interference. The simulations conducted using Castalia simulator show that by using a closed loop feedback system that the system can reduce the energy consumption of the nodes without compromising packet receive rates. Although similar to our work and the work in [7] the author in [8] designed a microcontroller to run the algorithm that contains the closed control loop. Our work differs for the reasons given previously, we are simulating energy in a network with a volatile topology.

The above is key and currently literature in relations to reducing the transmission power to save energy. Notwithstanding the different reasons for saving energy, it agrees with all research reviewed that saving energy is of great importance. Different approaches have been made to tackling this area of research, for example in [1] an empirical model was used, in [2] authors focused more on the characteristics of antennas and power consumption. In [6] authors were interested with determining the link quality and number of bits to transmit to determine the transmission power. In [7] the authors focused on two sensor networks and altered the transmission power and analyzed the performance of the network. Work published in [8] is like that of [7] but authors of [8] discuss a hardware implementation to their solution.

This research applies power saving techniques to a Mobile Ad Hoc Network (MANET) to prolong the lifetime of that network. A MANET is a packet switching data communications network that operates without a predefined infrastructure. The nodes within a MANET communicate using wireless technology and transmit radio waves omnidirectionally. These radio waves are received by all nodes within the transmission range of the sender. To communicate with a node that is not within the senders' transmission range, the nodes that are within range of the sender will forward the packet on behalf of the sender, therefore, a node within a MANET also takes on the additional responsibility of routing and forwarding packets.

There are many applications for a MANET which range from personal area networks (PANs) to large scale Wide Area Networks (WANs) that could potentially span over many miles [9]. There are also many application areas, for example, Vehicular ad hoc networks (VANETs) and Smart phone ad hoc networks (SPANs). The application that this research considers is that of Disaster rescue ad hoc networks [10]. Disaster recovery could be a result of a natural catastrophe, such as a landslide or sinkhole, or it could be because of a malicious attack such as a bomb, or there could have been some form of accident such as a fire. It is essential in these situations to establish a communications infrastructure with the intention to coordinate efforts which may potentially save lives.

Due to the critical application area, it is not only important to establish communications quickly, but it is also important to maintain communications. Due to the nature of the nodes being mobile, the nodes are normally equipped with a finite energy source, for example, a lithium battery. It is important for the network to be as efficient as possible in terms of energy depletion of the nodes.

Energy consumption in a MANET has been a focus of research in recent years $[11,13]$ and many authors have looked at ways to improve the power consumption by optimizing the performance of routing protocols $[12,14]$ or by managing the flow of packets using sliding windows and buffers [15]. Thus, much of the research in energy is conducted at the network layer.

This research optimizes energy consumption by using a cross-layered approach and reduces power-output at the physical layer by dynamically reducing the transmission and reception power of the antennas of the nodes. This approach was taken because of the broadcast nature of a MANET, that is, when a packet is sent all nodes within communications range of the source node will receive that packet and process the packet, even when they are not the intended recipient. In addition to this larger transmission areas could result in media contention issues and congestion which delays the sending of packets.

The theoretical design presented in this manuscript was implemented in Network Simulator 3 and consists of several simulations ranging from areas of low density to areas of high density. In all the simulations run the lifetime of the network was increased with little or no consequence in relation to packet delivery rate.

The remainder of this manuscript is organized as follows. In the Materials and Methods section, we define the simulation parameters with justification for these. The results section clearly shows a correlation between transmission/reception power and the life-time of the communications network, whilst still maintaining a quality of service. The discussion section details the findings of this research and offers insight for the results. The conclusion summaries the findings and discusses potential future work.

\section{Materials and Methods}

This section details the parameters used for the simulations that were conducted. To establish the effect of the lifetime and of the performance of the network two scenarios were devised. The first scenario consists of 9 nodes in a grid formation. Node 0 transmits a steady stream of packets to node 8 . In Scenario 2 the number of nodes is 
doubled to 18 , these are also placed in a grid formation. Scenario 2 also utilized multiple sources and destinations, node 0 transmits to node 14 and node 4 transmits to node 17. Each of the scenarios contained three types of configuration ( $a, b$ and $c)$, the first configuration (a), nodes were fixed and did not move position, the second configuration (b) the Random Waypoint Mobility Model (RWP) is applied, the third configuration (c) dynamically altered transmission power whilst the simulation was running. All simulations ran for $500 \mathrm{~s}$ except for configuration c, which ran for $504 \mathrm{~s}$. Table 1 summarizes the general configurations.

\begin{tabular}{|c|c|c|c|c|c|c|}
\hline & \multicolumn{6}{|c|}{ Scenarios } \\
\hline & $1 A$ & $1 B$ & $1 C$ & $2 A$ & $2 B$ & $2 C$ \\
\hline $\begin{array}{l}\text { Number of } \\
\text { Nodes }\end{array}$ & \multicolumn{3}{|c|}{9} & \multicolumn{3}{|c|}{18} \\
\hline $\begin{array}{l}\text { Number of } \\
\text { Power } \\
\text { Levels }\end{array}$ & \multicolumn{3}{|c|}{21} & \multicolumn{2}{|c|}{14} & 21 \\
\hline $\begin{array}{l}\text { Geographic } \\
\text { al Area }\end{array}$ & \multicolumn{3}{|c|}{$200 \times 200 \mathrm{~m}$} & \multicolumn{3}{|c|}{$500 \times 200 \mathrm{~m}$} \\
\hline $\begin{array}{l}\text { Mobility } \\
\text { Model }\end{array}$ & $\mathrm{C}$ & RWP & $\mathrm{C}$ & $\mathrm{C}$ & RWP & $\mathrm{C}$ \\
\hline $\begin{array}{l}\text { Simulation } \\
\text { Time }\end{array}$ & \multicolumn{2}{|c|}{$500 \mathrm{~s}$} & $504 \mathrm{~s}$ & \multicolumn{2}{|c|}{$500 \mathrm{~s}$} & $504 \mathrm{~s}$ \\
\hline
\end{tabular}

Table 1. Summary of the Scenarios ( $C$ refers to a Constant mobility model).

\subsection{Nodes}

In the context of MANET, a node is any device that has to capability of transmitting and receiving radio signals. In Scenario 1 the number of nodes participating in the network is 9, in Scenario 2 the number of nodes is doubled to 18 , see Figure 1 for an illustration of the increase in number of nodes for each scenario and the increase in geographical area, the image is a screen capture from Network Animator (NetAnim) and the nodes are represented by red circles. In Figure 1, the left topology shows a point in time before transmission has begun, the right topology shows transmission between the nodes is occurring and is represented by blue directional lines.

\subsection{Network Animators}

NetAnim (Network Animator) version 3.108, is bundled with Network Simulator 3 version 3.27, and is a sophisticated visualisation tool. The NetAnim graphical user interface allows the animation of packets propagating over both wired and wireless links. NetAnim also supports Visualisation for packet timelines, the ability to see the node position(s) at a given point in time (including node trajectory), plus more features including but not limited to routing tables at various times for the node(s).

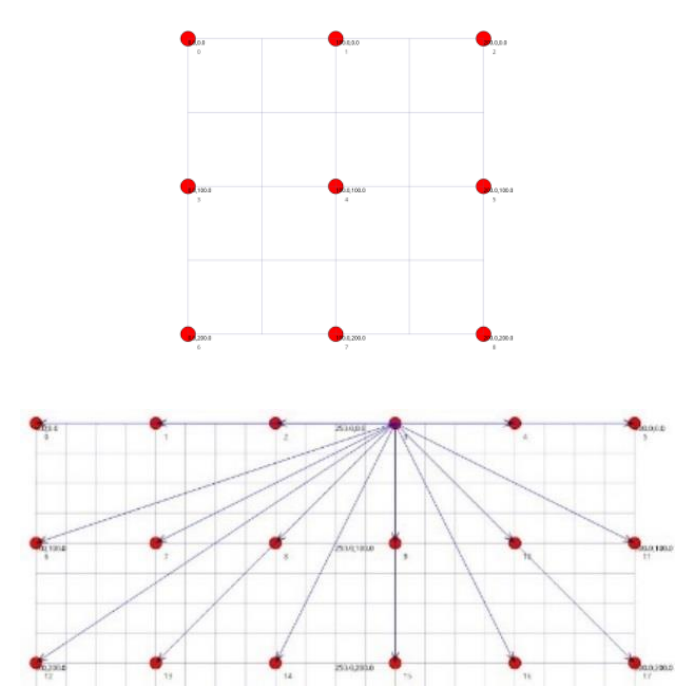

Figure 1. The $3 \times 3$ and $6 \times 3$ Network Topologies on the top and bottom panels, respectively.

\subsection{Antennas, Transmission and Energy Sources}

At the Physical (PHY) layer, the nodes were configured to use Direct Sequence Spread Spectrum (DSSS) at a rate of $11 \mathrm{Mbit} / \mathrm{s}$. At the application layer, the packet size was configured to 2048 bits with a data transmit rate of 2048 bit/s. Transmission mode for non-unicast data frames was also set to DSSS. The wireless standard used in the simulations is the IEEE 802.11b. A constant speed propagation delay model was applied with the default propagation speed of light in a vacuum measured in meters per second (m/s) or 299,792,458 m/s. The Friis Propagation Loss Model [16] was used to model propagation loss and is described by the formula:

$$
P_{r}=\frac{P_{t} \cdot G_{t} \cdot G_{r} \cdot \lambda^{2}}{(4 \cdot \pi \cdot d)^{2} \cdot L}
$$

Where $P_{r}$ is the reception power $(\mathrm{W}), P_{t}$ is the transmission power $(\mathrm{W}), G_{t}$ is the transmission gain (unitless), $G_{r}$ is the reception gain (unit-less), $\lambda$ is the wavelength $(\mathrm{m}), d$ is the distance $(\mathrm{m})$ and $L$ is the system loss (unit-less).

At the Media Access Control (MAC) layer the constant rate $\mathrm{Wi}-\mathrm{Fi}$ manager was disabled and the data mode and control mode assigned to the PHY layer. The MAC was also configured for operation in ad hoc mode.

The initial transmission power for the antennas of the nodes was set to $0.0 \mathrm{dBm}$, which is in line with current legislation which states transmission power should not exceed the threshold value of $20.0 \mathrm{dBm}$. Depending on the 
scenario being run the transmission power was set between $-10 \mathrm{~dB}$ and $+10 \mathrm{~dB}$. The transmission power remains constant until the transmission of the packet has ended. The real transmission power is calculated as follows:

$\underline{\text { TxPowerMin }+ \text { TxPowerLevel } \times \text { TxPowerMax }- \text { TxPowerMin }}$ number of TxLevels

The other power configuration settings can be found in Table 2.

\begin{tabular}{ll}
\hline \multicolumn{2}{c}{ Configuration Setting } \\
\hline Voltage & $3.0 \mathrm{~V}$ \\
$T_{x}$ Power Start/End & $-10 \mathrm{dBm}$ to $+10 \mathrm{dBm}$ \\
Number of $T_{x}$ Power Levels & $20($ steps of $1 \mathrm{~dB})$ \\
Idle Current & $0.273 \mathrm{~A}$ \\
$T_{x}$ Current & $0.389 \mathrm{~A}$ \\
\hline
\end{tabular}

Table 2. The transmission power settings

To transmit a packet, power must be drawn from an independent finite energy source of the node. In each of the simulations an energy source that represents a lithiumion battery was installed on each node. Lithium-ion batteries are common in mobile devices such as smart phones and laptops. The initial charge of every battery is 1000 Joules with a supply voltage of 3.0 Volts.

\subsection{Node Placement and Mobility Model}

The nodes were placed uniformly within the confines of the geographical area for the simulation run. Figure 1 shows initial placement of the nodes. The initial distance between each node for is 100 meters. The geographical area of Scenario 2 is double that of Scenario 1 to accommodate more nodes and the initial $100 \mathrm{~m}$ distance between the nodes.

Although the nodes were initially placed uniformly, their mobility consisted of random movements, speeds and pause times. The random waypoint mobility model was applied to each node for configurations $b$, therefore, Scenario $1 b$ and Scenario $2 b$ allowed the random movement of each node. Each node chooses a waypoint (next leg of journey) using a random value. A random speed is also chosen by each node for each leg of the journey, this was set between $0 \mathrm{~m} / \mathrm{s}$ and $2.5 \mathrm{~m} / \mathrm{s}$ (i.e. a maximum speed of $9 \mathrm{~km} / \mathrm{h}$ ) which is in line with the average walking speed. The pause speed (when arriving at each leg of the journey) was set to a constant of 0 seconds, thus the nodes moved continually for the duration of the simulation.

\subsection{Routing Protocols}

All nodes were configured with the Ad hoc On-Demand Distance Vector (AODV) routing protocol. AODV is a reactive routing protocol and scalable, global routing was enabled. All nodes interfaces (i.e. network cards) were configured with IP addresses starting at 10.1.1.1 - 10.1.1.n where $n$ is the number of nodes in the simulation +1 . The IP addresses were assigned sequentially. Thus, the node with ID 11 was assigned IP address 10.1.1.12 (node IDs start at 0 ). The subnet mask assigned was 255.255.255.0 which easily accommodated the number of nodes within the network.

\subsection{Traffic Flows}

Node 0 was configured to transmit packets to node 8 , beginning at $0 \mathrm{~s}$ with a constant random variable of 1.0, i.e. from some point between 0.0-1.0 s. Transmission of packets is persistent until the end of the simulation $(500 \mathrm{~s}$ or $504 \mathrm{~s}$ depending on configuration). The nodes used the user datagram protocol (UDP) as the transport protocol and transmitted at a constant bit rate (CBR) of $2048 \mathrm{~B} / \mathrm{s}$. A call back (trace) was configured at node 0 , which allowed the capturing of sent and received packets, further detail is provided in Section 2.8.

The above describes the traffic flow for Scenario 1 ( $a, b$ and $c$ ), for Scenario 2 ( $a, b$ and $c$ ) an additional traffic flow was configured between node 4 (sender) and node 17 (destination). The same configuration as discussed above was used for this additional traffic flow.

\subsection{Transmission and Received Power}

The transmission and reception power for configurations $\mathrm{a}$ and $\mathrm{b}$ for both Scenarios 1 and 2 is configured before the simulation begins and remains constant for the duration of the simulation. Thus, to evaluate network performance for 21 different transmission/reception power levels $(-10 \mathrm{~dB}$ to $+10 \mathrm{~dB}) 21$ distinct and separate simulations were conducted.

Scenario 1c and Scenario $2 c$ used dynamic transmission power levels, i.e. the transmission power dynamically changed as the simulation ran. The transmission power was set to change at intervals of 24 seconds with increments of $1 \mathrm{~dB}$ per interval. This is the reason that simulations performed with configuration $\mathrm{c}$ are slightly longer in duration than $500 \mathrm{~s}$.

\subsection{Data Acquisition}

Network Simulator 3 is a discrete network simulator and as such allows for the capture of many different types of data at many layers of the OSI model. This section details the data that is of interest for this research and the prelimnary simulations conducted to ensure that data is captured accurately. Network performance can be measured by packet delivery ratio (PDR), which is the number of received packets in relation to the number of sent packets, the network is deemed to be performing better with the higher percentage of sent packets being received. 
Also of interest is the power consumption of the nodes within the network, thus we needed to capture the data associated with the energy sources installed in the nodes. In particular for the nodes involved in the transmitting and receiving of data (the source and the destinations). This allows for analysis to determine if the network lifetime could be prolongated in relation to the transmission power values.

\subsubsection{Received Packets}

Each time a packet is received by the destination, a packet counter variable is updated by one. At each second of the simulation, throughput is calculated using number of packets captured for that duration. These calculations are stored in a Comma Separated Value (CSV) file. This file also contains the time at which the throughput was calculated, the number of packets received, the number of sinks, which routing protocol was used and the $T_{x}$ power used to transmit the packet. A snippet of the file is shown in Table 3.

\begin{tabular}{cccccc}
\hline $\begin{array}{c}\text { Time } \\
\text { Stamp }\end{array}$ & $\begin{array}{c}\text { Receive } \\
\text { Rate }\end{array}$ & $\begin{array}{c}\text { Received } \\
\text { Packets }\end{array}$ & $\begin{array}{c}\text { Number } \\
\text { of Sinks }\end{array}$ & $\begin{array}{c}\text { Routing } \\
\text { Protocol }\end{array}$ & $\begin{array}{c}\text { Transmission } \\
\text { Power }\end{array}$ \\
\hline 98.000 & 0.000 & 0.000 & 1 & AODV & 0.000 \\
99.000 & 0.000 & 0.000 & 1 & AODV & 0.500 \\
100.000 & 0.000 & 0.000 & 1 & AODV & 1.000 \\
101.000 & 10.752 & 72.000 & 1 & AODV & 1.500 \\
102.000 & 36.864 & 72.000 & 1 & AODV & 2.000 \\
103.000 & 36.864 & 72.000 & 1 & AODV & 2.500 \\
104.000 & 36.864 & 72.000 & 1 & AODV & 2.500 \\
118.000 & 36.864 & 72.000 & 1 & AODV & 2.500 \\
119.000 & 36.864 & 72.000 & 1 & AODV & 2.500 \\
\hline
\end{tabular}

Table 3. Received Packets according to time stamp, rate of reception, number of received packets and number of sinks $\left(1^{\text {st }}, 2^{\text {nd }}, 3^{\text {rd }}\right.$ and $4^{\text {th }}$ columns respectively).

\subsection{Transmitter State and Remaining Energy}

The transmission power is captured in $\mu$ s - microseconds and also when a change to transmission power had occurred, in addition the new power value was captured. To monitor the lifetime of the network we also captured the energy values (i.e. the remaining energy for the energy source) for each and every node, every $5 \mathrm{~s}$. All captured data were output to Comma Separated Value (CSV) text files for the analysis in MATLAB (The Mathworks Inc ${ }^{\circledR}$ ), R or other analytical software. The simulation also captured the state of the transmitter continually for each node within the simulation. Figure 2 gives an example of the transmitter state file for node 0 . The figure also shows the current simulator time in seconds, the state of the transmitter, when it entered this state and the duration of the state. This assured that the antenna model was operating correctly.

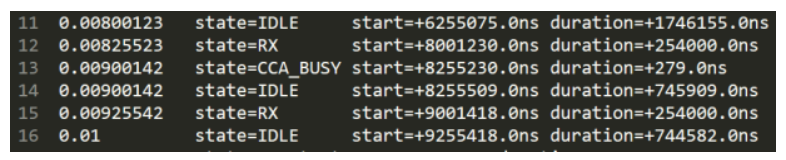

Figure 2. Acquisition of the Transmitter States.

The remaining energy for each node was also captured into a text file. All nodes started out with equal power for their energy sources. The draw on the energy source is dependent on what state the transmitter is in. For example, when in Idle state the current drawn is $0.273 \mathrm{~A}$, but when the transmitter is in $T_{x}$ (transmitting) state, the current drawn is $0.389 \mathrm{~A}$. The transmission power will influence the current drawn when in $T_{x}$ state, the higher the transmission power the greater the ampere drawn.

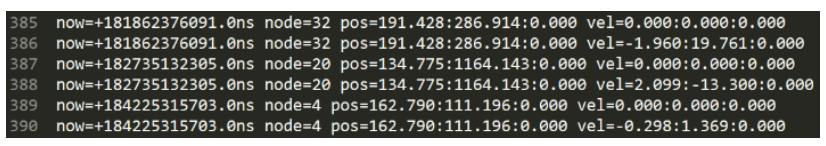

Figure 3. Acquisition of the Mobility States.

\subsection{Mobility}

The mobility of all the nodes for both Scenarios $1 b$ and $2 b$ was also captured. This data was captured because if something unexpected happened, the position of each of the nodes at a given moment in time could be accertained for analysis. The data captured is time, node identifier, the current position at that time and the velocity of the node. Figure 3 illustrates the format of the data captured. There are two lines per time entry, the second entry shows the nodes trajectory.

\subsection{Transmission Power \& Distance}

To ensure the simulations were running as expected many preliminary simulations were run, 41 of the preliminary simulations that were run each used a different transmission power setting. Each of these simulations consisted of only two nodes which began sufficiently close to one another so that they could communicate (in transmission range). At each second, node 1 would move one meter away from node 0 , node 1 continued to do this until communication ceased, this gave a maximum distance for a given transmission power. Table 4 shows the results from these preliminary simulations.

\begin{tabular}{cccc}
\hline $\boldsymbol{T}_{\boldsymbol{x}}$ Power & Distance $_{\text {Max }}$ & $\boldsymbol{T}_{\boldsymbol{x} \text { Power }}$ & Distance $_{\text {Max }}$ \\
\hline-20 & 12 & 1 & 151 \\
-19 & 14 & 2 & 168 \\
-18 & 16 & 3 & 191 \\
-17 & 18 & 4 & 215 \\
-16 & 20 & 5 & 241
\end{tabular}




\begin{tabular}{cccc}
-15 & 23 & 6 & 270 \\
-14 & 25 & 7 & 304 \\
-13 & 30 & 8 & 342 \\
-12 & 33 & 9 & 382 \\
-11 & 38 & 10 & 430 \\
-10 & 42 & 11 & 481 \\
-9 & 47 & 12 & 539 \\
-8 & 52 & 13 & 605 \\
-7 & 59 & 14 & 678 \\
-6 & 66 & 15 & 763 \\
-5 & 75 & 16 & 864 \\
-4 & 85 & 17 & 967 \\
-3 & 96 & 18 & 1084 \\
-2 & 107 & 19 & 1215 \\
-1 & 121 & 20 & 1355 \\
0 & 134 & & \\
\hline
\end{tabular}

Table 4. The simulation results: $T_{x}$ Power vs Distance $_{\text {Max. }}$

Figure 4 depicts the plotted data from Table 4 and shows that nodes can successfully communicate over longer distances by increasing the transmission power.

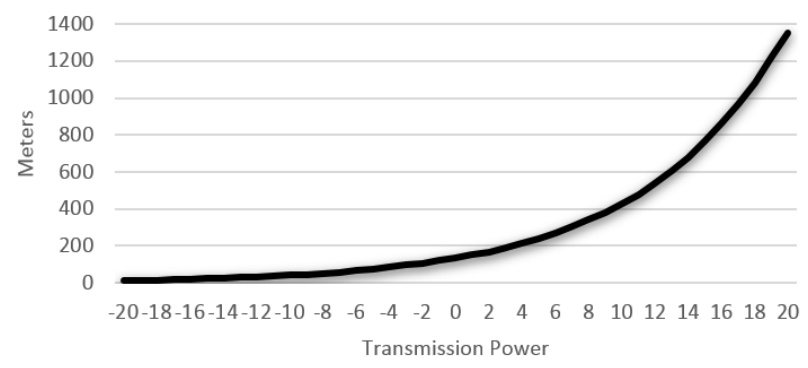

Figure 4. Transmission Power vs Distance.

Other preliminary simulations allowed the checking of energy sources to ensure that they were being depleted correctly, when an energy source dropped to level that was below a transmission/reception energy threshold the node entered a sleep state and could not transmit or receive further packets.

In addition, traffic flows were checked to ensure they worked as expected and packets were captured correctly. NetAnim also helped verify some other configuration settings such as node placement and mobility. In addition to this live output was observed from the simulator as it ran, this output consisted of all the changes or events of interest that occurred in the simulation.

See Figure 5 which is a capture of an area of the Linux terminal window and depicts the beginning of a simulation run.

\subsection{Data Captured for Scenarios 1 and 2}

Once convinced by the preliminary simulations that the network was running and capturing data accurately we tailored the data captured. Data was captured continuously as the simulation ran which resulted in huge data sets. Therefore, because of computational resources we summarized the continuously captured data and output the results to a CSV file every $5 \mathrm{~s}$. Namely the data of interest were; simulation time, the number of sent packets, the number of received packets, the energy depleted, the transmission power, the position of the nodes and the distances between the nodes.

Other data, as described earlier was also captured for further verification but was not used as part of the main analysis, although it provided useful when discussing the analysis to confirm the assumptions made were justified and correct, for example, NetAnim was also configured to capture the animator XML file.

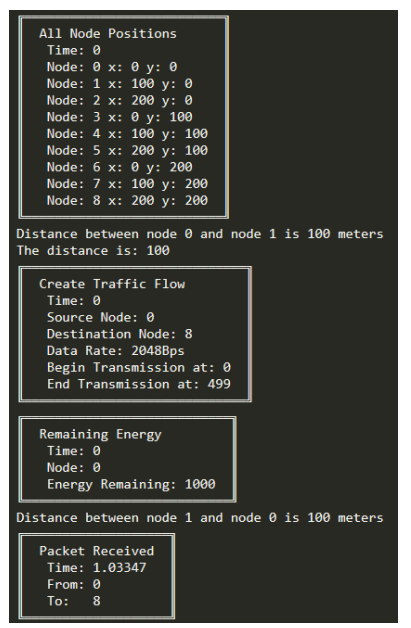

Figure 5. Live output as the simulation runs.

\section{Results}

Each one of the two scenarios were simulated in three different configurations, namely configuration a, configuration $\mathrm{b}$ and configuration $\mathrm{c}$.

Configuration a used a grid placement model and a constant mobility model (the nodes did not move from their original placement positions). Configuration $b$ was $a$ variation of configuration a, but the random waypoint mobility model was applied with restrictive mobility within the original defined geographical areas (Scenario 1, $200 \mathrm{~m}$ x $200 \mathrm{~m}$ and Scenario 2, $500 \mathrm{~m}$ x $200 \mathrm{~m}$ ). Configuration a and $b$ were run many times, once for each transmission power level being evaluated. In Scenario 1, transmission power was evaluated from $-10 \mathrm{~dB}$ to $+10 \mathrm{~dB}$ in steps of 1 $\mathrm{dB}$, thus a total of 21 simulations for each configuration were run. In Scenario 2, the transmission power was evaluated from $-3 \mathrm{~dB}$ to $+10 \mathrm{~dB}$, thus for each configuration a and $\mathrm{b}$, a total of 14 simulations were run.

Configuration $c$ was quite different because the transmission power was altered during the simulation every 
24 seconds, starting at $-10 \mathrm{~dB}$ and incrementing in steps of $1 \mathrm{~dB}$ to $+10 \mathrm{~dB}$. Thus, a range of transmission power levels were tested in two simulations.

In total 72 unique and independent simulations were run, and the data captured (number of sent packets, number of received packets, energy spent, energy remaining, etc.) analysed to determine the most optimal transmission power for a 'greener' network. Table 5 presents a summary of the simulations.

\begin{tabular}{ll}
\hline & Simulations \\
\hline Scenario $1 a$ & 21 simulations (Constant Mobility - CM) \\
Scenario $1 b$ & 21 simulations (Random Waypoint \\
& Mobility - RWM) \\
Scenario $1 c$ & 1 simulation (CM) \\
Scenario $2 a$ & 14 simulations (CM) \\
Scenario $2 b$ & 14 simulations (RWM) \\
Scenario $2 c$ & 1 simulation (CM) \\
\hline
\end{tabular}

Table 5. Overview of the overall simulations.

\subsection{Scenario $1 \mathrm{a}$}

Scenario la consisted of nine nodes in a grid placement formation, see Figure 1, the nodes are placed $100 \mathrm{~m}$ apart and have a constant mobility model applied, i.e. the nodes do not move. Node 0 is transmitting UDP packets to node 8 at approximately one packet per second, see Table 6. A total of 21 simulations were performed with the only varying parameter being the transmission power. Data is captured every $5 \mathrm{~s}$ with a total simulation time of 500 seconds

\begin{tabular}{ll}
\hline \multicolumn{2}{c}{ Scenario 1a } \\
\hline Placement Model & $3 \times 3$ Grid \\
Distance between Nodes & 100 Meters \\
Mobility Model & Constant \\
Transport Protocol & UDP \\
Data Rate & $2048 \mathrm{~B} / \mathrm{s}$ \\
Packet Size & $2048 \mathrm{Bytes}$ \\
Transmission Power & $-10 \mathrm{~dB}$ to $+10 \mathrm{~dB}$ \\
Data Capture Interval & 5 seconds \\
Simulation Duration & 500 seconds \\
\hline
\end{tabular}

Table 6. Setting under Scenario $1 a$.

When the transmission power was set between $-10 \mathrm{~dB}$ and $-3 \mathrm{~dB}$ (i.e. in the first 8 simulation runs) no packets were received by node 8 . This might not be too surprising since the transmission power was set relatively low. However, since we are interested in energy depletion, we need to test the situation when the source and destination did not have higher enough transmission power to successfully send or receive packets over 100 meters.

Figure 6 depicts the depletion of energy at Node 0 , initially the energy was set to 1000 Joules and depleted to 594.59 Joules. The power depletion of both node 0 (sender) and node 8 (receiver) showed a linear decline which was identical between both nodes, this decline is the same for transmission power between $-10 \mathrm{~dB}$ and $-5 \mathrm{~dB}$. This decline is approximately $-40.6 \%$ for $500 \mathrm{~s}$ (Figure 6).

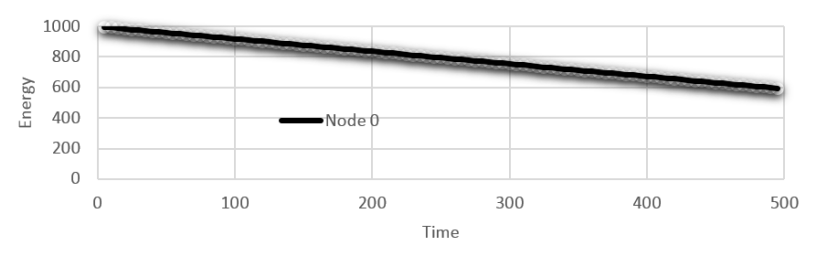
Figure 6. Energy Depletion and Transmission Power
of Scenario 1a.

Packets begin to be received when transmission power is set to $-2 \mathrm{~dB}$, however, network performance is poor with approximately $79 \%$ of transmissions being unsuccessful, 494 packets were sent and 103 were successfully received (Figure 7). Power consumption is comparable to the earlier simulation runs with a decline of approximately $-40.7 \%$.

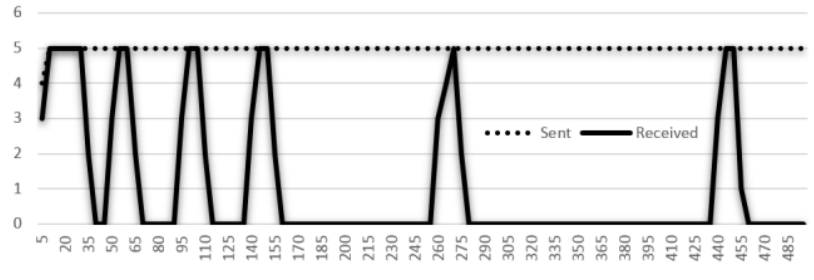

Figure 7. Packets Sent vs Packets Received at Transmission Power -2dB.

With a higher transmission power of $-1 \mathrm{~dB}$ one would expect an increase in performance, however, this yielded zero successfully received packets with power consumption like that of the previous simulation runs. When the transmission power is set to $0 \mathrm{~dB}$ packets begin to be received again but performance of the network is very poor with approximately $96 \%$ of packets not received.

Higher transmission power levels $(+1 \mathrm{~dB}$ to $+10 \mathrm{~dB})$ show better performance with a higher number of packets being received, most notably $+7 \mathrm{~dB}$ to $+10 \mathrm{~dB}$ show near perfect performance. There are fluctuations in performance for transmission power levels of $+2 \mathrm{~dB}$ to $+6 \mathrm{~dB}$ with a notable decline in the number of received packets for the power level $+6 \mathrm{~dB}$. Table 7 summarises the number of packets sent and received as well as the energy remaining for the sender and the receiver. This is plotted and shown in Figure 8.

\begin{tabular}{ccccc}
\hline & \multicolumn{2}{c}{ Packets } & \multicolumn{2}{c}{ Energy Remaining } \\
\hline $\boldsymbol{T}_{\boldsymbol{x} \text { Power }}$ & Sent & Received & Sender & Receiver \\
\hline-10 & 494 & 0 & 594.592 & 594.592 \\
-9 & 494 & 0 & 594.592 & 594.592 \\
\hline
\end{tabular}




\begin{tabular}{ccccc}
\hline-8 & 494 & 0 & 594.590 & 594.590 \\
-7 & 494 & 0 & 594.588 & 594.588 \\
-6 & 494 & 0 & 594.587 & 594.587 \\
-5 & 494 & 0 & 594.487 & 594.470 \\
-4 & 494 & 0 & 593.855 & 593.896 \\
-3 & 494 & 0 & 593.504 & 593.738 \\
-2 & 494 & 103 & 593.393 & 593.570 \\
-1 & 494 & 0 & 593.214 & 593.665 \\
0 & 494 & 22 & 592.735 & 593.708 \\
1 & 494 & 474 & 593.609 & 594.047 \\
2 & 494 & 330 & 593.228 & 594.013 \\
3 & 494 & 253 & 592.798 & 593.924 \\
4 & 494 & 154 & 591.694 & 593.899 \\
5 & 494 & 178 & 590.071 & 593.817 \\
6 & 494 & 48 & 602.184 & 608.570 \\
7 & 494 & 492 & 660.485 & 662.194 \\
8 & 494 & 493 & 710.640 & 711.660 \\
9 & 494 & 493 & 755.417 & 756.503 \\
10 & 494 & 493 & 795.185 & 796.342 \\
\hline
\end{tabular}

Table 7. Packets and Remaining Energy (Scenario 1a).

Figure 8 shows the energy remaining for the source and destination nodes and is comparable (upper line of the graph), this upper line shows that the higher the transmission power the less energy is consumed. The lower line of the graph shows the number of packets received for each of the transmission power settings, this shows that higher transmission power yields more received packets but not at the expense of power consumption. A similar number of packets were received when transmitting on a lower power $(1 \mathrm{~dB})$, however, the power consumption is greater.

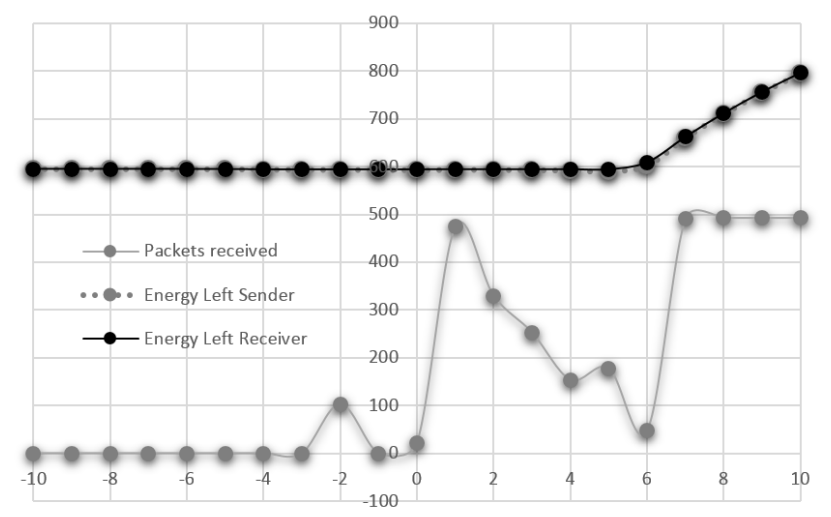

Figure 8. Energy left and received packets vs the transmission power in Scenario $1 a$.

Figure 9 illustrates the power consumption for the sender and the receiver based on the transmission power setting once the simulations had been completed (i.e. at time equal to $500 \mathrm{~s}$ ). Clearly the power consumption is fairly uniform for transmission power setting $-10 \mathrm{~dB}$ to +5
$\mathrm{dB}$, however, from $+6 \mathrm{~dB}$ to $+10 \mathrm{~dB}$ each higher transmission setting conserves more power.

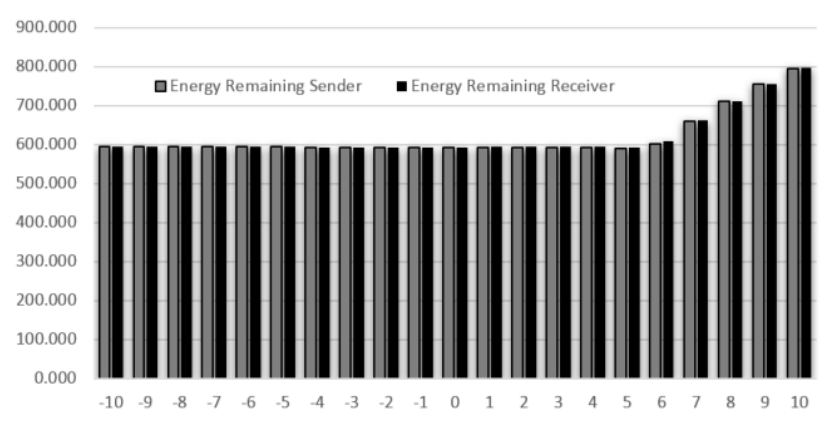

Figure 9. Energy Remaining after 500 seconds for Transmission Powers $-10 \mathrm{~dB}$ to $+10 \mathrm{~dB}$.

\subsection{Scenario $1 b$}

Scenario $1 b$ is an adaption of Scenario 1 a except for the constant mobility model, since the Random Waypoint Mobility Model (RWM) was applied (Table 8). When applying the RWM, we did not want the nodes to wander outside of the boundaries of the original defined world (200 $\mathrm{m} \times 200 \mathrm{~m}$ ), therefore maximum $x$ and $y$ positional values for each node were set to 200, which kept the mobility of the node constrained within the original area defined. The RWM assigns points of the journey called waypoints, each node selects a speed between $0 \mathrm{~m} / \mathrm{s}$ and $20 \mathrm{~m} / \mathrm{s}$ and heads in a straight line from its current position to the waypoint. This process is repeated for each waypoint. There is the option for the node to pause at each waypoint but in this model the waypoint delay was set to 0 , thus the nodes continually moved for the duration of the simulation.

\begin{tabular}{ll}
\hline \multicolumn{2}{c}{ Scenario $\mathbf{1 b}$} \\
\hline Placement Model & $3 \times 3$ Grid \\
Distance between Nodes & 100 Meters \\
Mobility Model & Random Waypoint \\
Maximum X Value & 200 \\
Maximum Y Value & 200 \\
Minimum Node Speed & $0 \mathrm{~m} / \mathrm{s}$ \\
Maximum Node Speed & $20 \mathrm{~m} / \mathrm{s}$ \\
Waypoint Delay & $0 \mathrm{~s}$ \\
Transport Protocol & $\mathrm{UDP}$ \\
Data Rate & $2048 \mathrm{~B} / \mathrm{s}$ \\
Packet Size & $2048 \mathrm{Bytes}$ \\
Transmission Power & $-10 \mathrm{~dB}$ to $+10 \mathrm{~dB}$ \\
Data Capture Interval & $5 \mathrm{~s}$ \\
Simulation Duration & $500 \mathrm{~s}$ \\
\hline
\end{tabular}

Table 8. Setting under Scenario $1 b$.

The results are somewhat similar to Scenario la, however, the noticeable differences are that there is no period when packets are not being received. Moreover, the number of received packets has almost a linear relationship 
with the transmission power, except for the case when the transmission power is set to $-1 \mathrm{~dB}$ and a drop in the number of received packets occurs. Observed in Scenario $1 a$ is the degrading performance when transmission power was set between $+2 \mathrm{~dB}$ and $+6 \mathrm{~dB}$, such a decline has not resulted in this scenario. Figure 8 depicts the results: the $x$ axis reports the transmission power and the $y$ axis shows either the number of received packets or the energy remaining, depending on context.

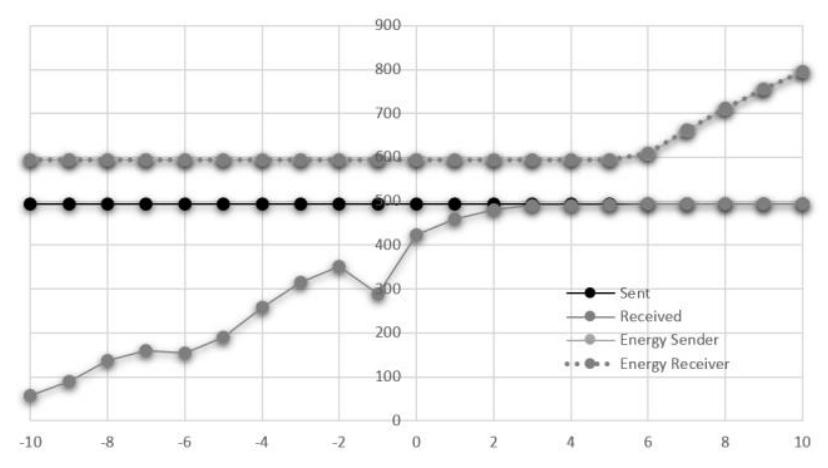

Figure 10. Remaining Energy vs Transmission Power under Scenario $1 b$.

The relationship between transmission power and remaining energy is almost identical to that in Scenario 1a with fractional deviations. Table 9 presents sent and received packets statistics and energy remaining for each of the simulations conducted.

\begin{tabular}{ccccc}
\hline & \multicolumn{2}{c}{ Packets } & \multicolumn{2}{c}{ Energy Remaining } \\
\hline $\boldsymbol{T}_{\boldsymbol{x} \text { Power }}$ & Sent & Received & Sender & Receiver \\
\hline-10 & 494 & 57 & 594.352 & 594.052 \\
-9 & 494 & 90 & 594.312 & 594.024 \\
-8 & 494 & 138 & 594.280 & 594.050 \\
-7 & 494 & 160 & 594.172 & 593.974 \\
-6 & 494 & 155 & 594.105 & 593.963 \\
-5 & 494 & 190 & 594.070 & 594.008 \\
-4 & 494 & 259 & 594.047 & 594.068 \\
-3 & 494 & 315 & 594.036 & 594.129 \\
-2 & 494 & 351 & 593.965 & 594.137 \\
-1 & 494 & 289 & 594.058 & 594.270 \\
0 & 494 & 423 & 593.956 & 594.246 \\
1 & 494 & 460 & 593.969 & 594.277 \\
2 & 494 & 480 & 593.928 & 594.282 \\
3 & 494 & 491 & 593.871 & 594.283 \\
4 & 494 & 491 & 593.725 & 594.247 \\
5 & 494 & 492 & 593.529 & 594.224 \\
6 & 494 & 493 & 608.175 & 609.013 \\
7 & 494 & 493 & 661.314 & 662.244 \\
8 & 494 & 493 & 710.637 & 711.655 \\
9 & 494 & 493 & 755.391 & 756.492 \\
10 & 494 & 493 & 795.159 & 796.331 \\
\hline
\end{tabular}

Table 9. Packets and Remaining Energy (Scenario 1b).

\subsection{Scenario $1 \mathrm{c}$}

The final variation of Scenario 1 was to implement dynamic transmission power (Scenario 1c). In Scenario 1a and Scenario $1 b$ the simulations ran for 500 seconds on a fixed transmission power, thus with 21 transmission power settings, 21 simulations were run each for $500 \mathrm{~s}$. In this final scenario the interest was to determine how energy depletion and performance would be impacted if transmission power was changing whilst the simulation was running or 'on the fly'. Therefore, Scenario $1 c$ consisted of a single simulation with varying transmission power. The transmission power was increased every $24 \mathrm{~s}$ and begins at $-10 \mathrm{~dB}$ incrementing to $+10 \mathrm{~dB}$. Table 10 reminds the reader of the scenario settings with the additional information regarding the duration of the increase time of transmission power.

\begin{tabular}{ll}
\hline \multicolumn{1}{c}{ Scenario 1c } \\
\hline Placement Model & $3 \times 3$ Grid \\
Distance between Nodes & 100 Meters \\
Mobility Model & Constant \\
Transport Protocol & UDP \\
Data Rate & $2048 \mathrm{~B} / \mathrm{s}$ \\
Packet Size & $2048 \mathrm{Bytes}$ \\
Transmission Power & $-10 \mathrm{~dB}$ to $+10 \mathrm{~dB}$ \\
Transmission Power Increment & $24 \mathrm{~s}$ \\
Data Capture Interval & $5 \mathrm{~s}$ \\
Simulation Duration & $500 \mathrm{~s}$ \\
\hline
\end{tabular}

Table 10. Setting under Scenario 1c.

Table 11 presents a summary of the data every $24 \mathrm{~s}$ and Figure 11 shows the number of packets sent and received with the energy spent at the sender and the receiver for each transmission power setting.

\begin{tabular}{cccccccc}
\hline \multicolumn{9}{c}{ Packets } & \multicolumn{2}{c}{ Energy Remaining } & \multicolumn{2}{c}{ Energy Spent } \\
\hline Tim & TxPowe & $\begin{array}{c}\text { Sen } \\
\text { t }\end{array}$ & $\begin{array}{c}\text { Receive } \\
\text { d }\end{array}$ & Sender & $\begin{array}{c}\text { Receive } \\
\text { r }\end{array}$ & $\begin{array}{c}\text { Sende } \\
\text { r }\end{array}$ & $\begin{array}{c}\text { Receive } \\
\text { r }\end{array}$ \\
\hline 24 & -10 & 23 & 0 & 980.344 & 980.344 & 19.656 & 19.656 \\
48 & -9 & 24 & 0 & 960.688 & 960.688 & 19.656 & 19.656 \\
72 & -8 & 24 & 0 & 941.031 & 941.031 & 19.657 & 19.657 \\
96 & -7 & 24 & 0 & 921.375 & 921.375 & 19.656 & 19.656 \\
120 & -6 & 24 & 0 & 901.719 & 901.719 & 19.656 & 19.656 \\
144 & -5 & 24 & 0 & 882.062 & 882.062 & 19.657 & 19.657 \\
168 & -4 & 24 & 0 & 862.368 & 862.370 & 19.694 & 19.692 \\
192 & -3 & 24 & 0 & 842.657 & 842.670 & 19.711 & 19.700 \\
216 & -2 & 24 & 4 & 822.937 & 822.968 & 19.720 & 19.702 \\
240 & -1 & 24 & 0 & 803.230 & 803.267 & 19.707 & 19.701 \\
264 & 0 & 24 & 1 & 783.519 & 783.564 & 19.711 & 19.703 \\
288 & 1 & 24 & 22 & 763.819 & 763.880 & 19.700 & 19.684 \\
312 & 2 & 24 & 10 & 744.054 & 744.192 & 19.765 & 19.688 \\
336 & 3 & 24 & 5 & 724.266 & 724.487 & 19.788 & 19.705 \\
360 & 4 & 24 & 2 & 704.442 & 704.791 & 19.824 & 19.696 \\
384 & 5 & 24 & 22 & 684.695 & 685.105 & 19.747 & 19.686 \\
408 & 6 & 24 & 10 & 664.761 & 665.408 & 19.934 & 19.697 \\
432 & 7 & 24 & 23 & 644.987 & 645.731 & 19.774 & 19.677 \\
456 & 8 & 24 & 24 & 625.230 & 626.044 & 19.757 & 19.687 \\
480 & 9 & 24 & 24 & 605.456 & 606.361 & 19.774 & 19.683 \\
504 & 10 & 24 & 24 & 585.645 & 586.663 & 19.811 & 19.698 \\
\hline
\end{tabular}

Table 11. Summary of the results under Scenario $1 c$. 
Compared with the 21 simulations run in Scenario $1 a$ the results are quite similar, those similarities are:

- $\quad$ whilst the power was set between $-10 \mathrm{~dB}$ and -3 $\mathrm{dB}$ no packets were received

- at $-2 \mathrm{~dB}$, some packets were received but the network performance is poor

- at $-1 \mathrm{~dB}$, no packets were received

- at $0 \mathrm{~dB}$, packets began to be received but very few of them

- at $1 \mathrm{~dB}$, the performance increased significantly

- at $2 \mathrm{~dB}$, the performance has declined and continues to decline for each increase in the transmission power until a $7 \mathrm{~dB}$ value is reached

- $\quad$ at $7 \mathrm{~dB}$, the performance is near perfect

- at $8 \mathrm{~dB}$, until $10 \mathrm{~dB}$, all the transmitted packets are received.

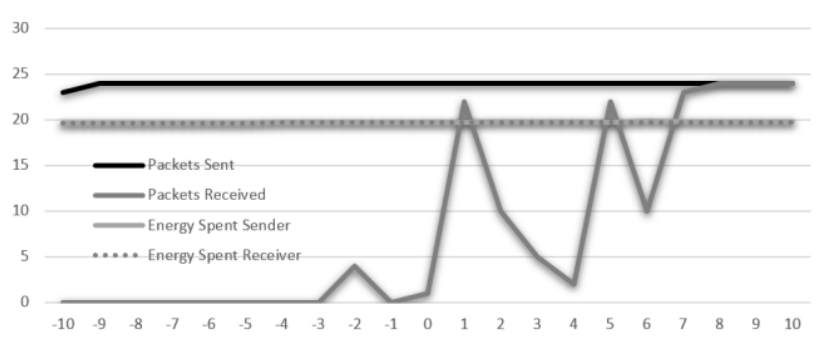

Figure 11. Remaining Energy vs Transmission Power under Scenario 1c.

Regarding the transmission power, this is comparatively similar for each transmission power, however, it should be noted that each transmission power was constant for only 24 s. The previous simulations have shown that transmission power is related to energy spent and the results show that the higher the transmission power the less energy is spent.

\subsection{Scenario $2 a$}

Scenario $2 a$ consisted of 18 nodes in a horizontal grid formation of $6 \times 3$ nodes which is depicted in Figure 1. The nodes are placed $100 \mathrm{~m}$ apart. Node 0 is transmitting UDP packets to Node 14 and Node 3 is transmitting packets to Node 17. The timing of the transmissions has been synchronised, i.e. both Node 0 and Node 3 are scheduled to transmit to their respective destinations at the same time. Node 0 and Node 3 are transmitting UDP packets at approximately one packet per second.

In this scenario the interest was to determine how transmission power from multiple sources may cause interference and thus influence performance - a lower transmission power should cause less interference within the geographical area. The only varying parameter for the simulation runs in this scenario is the transmission power so that comparisons from Scenario la can be drawn.
Unlike Scenario 1, 14 simulation runs were conducted with transmission power ranging from $-3 \mathrm{~dB}$ to $+10 \mathrm{~dB}$ because it had been established from Scenario 1 that a transmission power less than $-3 \mathrm{~dB}$ yields no received transmitted packets. Tables 12 and 13 present a summary of the results.

\begin{tabular}{ccccc}
\hline \multicolumn{5}{c}{ Node 0 transmitting to Node 14 } \\
\hline \multicolumn{4}{c}{ Packets } & \multicolumn{2}{c}{ Energy Remaining } \\
\hline $\boldsymbol{T}_{\boldsymbol{x} \text { Power }}$ & Sent & Received & Sender & Receiver \\
\hline-3 & 494 & 0 & 592.850 & 593.104 \\
-2 & 494 & 57 & 592.697 & 592.860 \\
-1 & 494 & 15 & 592.690 & 593.016 \\
0 & 494 & 25 & 592.132 & 592.881 \\
1 & 494 & 401 & 593.105 & 593.431 \\
2 & 494 & 300 & 593.211 & 593.553 \\
3 & 494 & 179 & 592.162 & 593.275 \\
4 & 494 & 2 & 590.387 & 593.077 \\
5 & 494 & 0 & 588.217 & 593.142 \\
6 & 494 & 36 & 602.318 & 607.877 \\
7 & 494 & 489 & 660.024 & 661.796 \\
8 & 494 & 494 & 710.387 & 711.421 \\
9 & 494 & 494 & 755.151 & 756.280 \\
10 & 494 & 494 & 794.963 & 796.172 \\
\hline
\end{tabular}

Table 12. Summary of the results under Scenario $2 a$ (Node 0 to Node 14).

\begin{tabular}{|c|c|c|c|c|}
\hline \multicolumn{5}{|c|}{ Node 3 transmitting to Node 17} \\
\hline & \multicolumn{2}{|c|}{ Packets } & \multicolumn{2}{|c|}{ Energy Remaining } \\
\hline$T_{x}$ Power & Sent & Received & Sender & Receiver \\
\hline-3 & 494 & 0 & 592.778 & 593.766 \\
\hline-2 & 494 & 172 & 592.586 & 593.317 \\
\hline-1 & 494 & 0 & 592.775 & 593.408 \\
\hline 0 & 494 & 28 & 592.171 & 593.347 \\
\hline 1 & 494 & 430 & 593.048 & 593.422 \\
\hline 2 & 494 & 269 & 593.010 & 593.552 \\
\hline 3 & 494 & 149 & 592.314 & 593.277 \\
\hline 4 & 494 & 99 & 590.208 & 593.052 \\
\hline 5 & 494 & 0 & 590.516 & 593.127 \\
\hline 6 & 494 & 33 & 602.300 & 607.864 \\
\hline 7 & 494 & 491 & 659.923 & 661.790 \\
\hline 8 & 494 & 494 & 710.398 & 711.398 \\
\hline 9 & 494 & 494 & 755.180 & 756.254 \\
\hline 10 & 494 & 494 & 794.986 & 796.143 \\
\hline
\end{tabular}

Table 13. Summary of the results under Scenario $2 a$ (Node 3 to Node 17).

When transmission power was set to $-3 \mathrm{~dB}$ no packets were received by neither destination, which is comparable with Scenario $1 a$, in addition to this the energy remaining at each node is also comparable. At $-2 \mathrm{~dB}$ packets are successfully received by both destinations but performance is relatively poor with $88 \%$ and $65 \%$ packet loss, which is comparable with Scenario 1a, which experienced $79 \%$ packet loss.

Network performance degraded even further when transmission power was set to $-1 \mathrm{~dB}$, in the case of node 17 , no packets at all are received which is the same as Scenario $1 a$, however node 14 did receive approximately 3 $\%$ of the packets that were sent. Increasing transmission 
power to 0 resulted in an increase in performance but this was only a slight increase. When the transmission power was set to $1 \mathrm{~dB}$ performance increased significantly with node 14 receiving approximately $81 \%$ of packets and node 17 receiving $87 \%$ of packets.

Increasing the transmission power to $2 \mathrm{~dB}$ performance dropped, as the power increased $(+3 \mathrm{~dB},+4 \mathrm{~dB}$ and $+5 \mathrm{~dB})$ performance dropped in an almost linear fashion until no packets were received by either destination. Notably, energy consumption from $-3 \mathrm{~dB}$ to $+5 \mathrm{~dB}$ remains almost constant, this is depicted in Figure 12 and shown by the upper line.

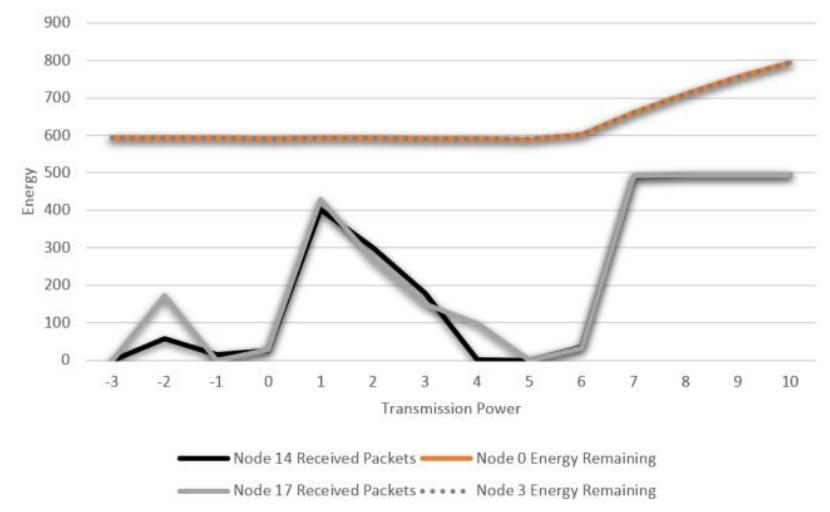

Figure 12. Remaining Energy and Packets Sent/Received vs Transmission Power under Scenario $2 a$.

Also depicted in Figure 12 is a slight increase in performance when increasing the transmission power from $5 \mathrm{~dB}$ to $6 \mathrm{~dB}$. At transmission powers $7 \mathrm{~dB}$ to $10 \mathrm{~dB}$ performance is excellent, at $7 \mathrm{~dB}$ less than $1 \%$ of packets are not received whist from $8 \mathrm{~dB}$ to $10 \mathrm{~dB}$ all sent packets are received. Notably, as transmission power is increased power consumption is decreased in a linear fashion.

\subsection{Scenario $2 b$}

Scenario $2 b$ is an adaption of Scenario $2 a$ except rather than a constant mobility model the RWM was applied (Table 14). When applying the RWM, we did not want the nodes to wander outside of the boundaries of the original defined world (500 m x $200 \mathrm{~m}$ ), therefore maximum $\mathrm{x}$ and y positional values for each node were set to 500 and 200 respectively. This kept the mobility of the node constrained within the original area defined. The RWM assigns points of the journey called waypoints, each node selects a speed between $0 \mathrm{~m} / \mathrm{s}$ and $20 \mathrm{~m} / \mathrm{s}$ and heads in a straight line from its current position to the waypoint. This process is repeated for each waypoint. There is the option for the node to pause at each waypoint but in this model the waypoint delay was set to 0 , thus the nodes continually moved for the duration of the simulation.

\begin{tabular}{ll}
\hline \multicolumn{2}{c}{ Scenario $2 \boldsymbol{b}$} \\
\hline Placement Model & $6 \times 3$ Grid \\
Distance between Nodes & 100 Meters \\
Mobility Model & Random Waypoint \\
Maximum X Value & 500 \\
Maximum Y Value & 200 \\
Minimum Node Speed & $0 \mathrm{~m} / \mathrm{s}$ \\
Maximum Node Speed & $20 \mathrm{~m} / \mathrm{s}$ \\
Waypoint Delay & $0 \mathrm{~s}$ \\
Transport Protocol & $\mathrm{UDP}$ \\
Data Rate & $2048 \mathrm{~B} / \mathrm{s}$ \\
Packet Size & $2048 \mathrm{Bytes}$ \\
Transmission Power & $-3 \mathrm{~dB}$ to $+10 \mathrm{~dB}$ \\
Data Capture Interval & $5 \mathrm{~s}$ \\
Simulation Duration & $500 \mathrm{~s}$ \\
\hline
\end{tabular}

Table 14. Setting under Scenario $2 b$.

When the RWM is applied there are no periods when packets are not received for all transmission powers simulated $(-3 \mathrm{~dB}$ to $10 \mathrm{~dB})$. As one might expect performance at $-3 \mathrm{~dB}$ was poor and $81.38 \%$ of packets were not received by node 14 . Node 17 received slightly more packets, but the loss was still $74.90 \%$. As transmission increased there was an increase in performance for both destinations $(-2 \mathrm{~dB}$ to $0 \mathrm{~dB})$. Node 14 received slightly fewer packets when transmission power was changed from $0 \mathrm{db}$ to $1 \mathrm{~dB}$, but this is only $1.49 \%$ fewer packets and is negligible. When transmission power increased from $1 \mathrm{~dB}$ to $2 \mathrm{~dB}$, Node 14 received more packets, however, this dropped again when transmission power was set to $3 \mathrm{~dB}$. At $3 \mathrm{~dB}$, Node 14 received $27 \%$ fewer packet than when it was set to $2 \mathrm{~dB}$. Node 14 then began to receive more and more packets as the transmission power was increased, however, it was not until transmission power was at $5 \mathrm{~dB}$ that more packets were received than when transmission power was set to $2 \mathrm{~dB}$. At $6 \mathrm{~dB}$, Node 14 saw a relatively sharp increase in performance, then from $7 \mathrm{~dB}$ to $10 \mathrm{~dB}$ perfomance increased but more gradually.

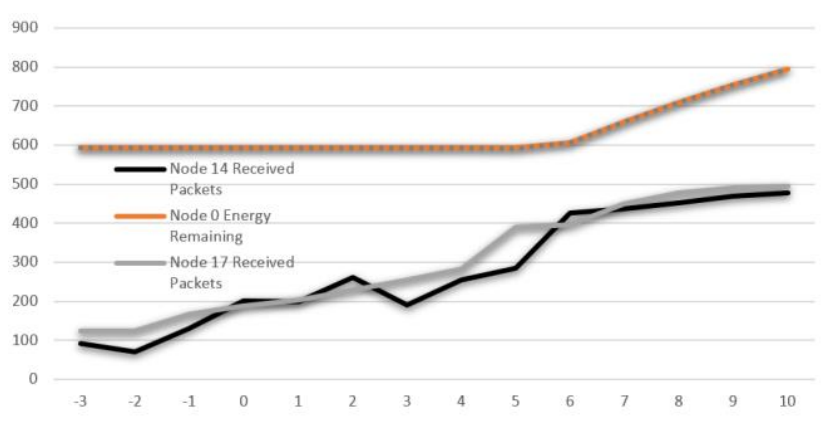

Figure 13. Remaining Energy and Packets Sent/Received vs Transmission Power under Scenario $2 b$. 
The number of received packets for Node 17 showed no decline when transmission power was increased, as the transmission power increased so did the number of received packets.

Somewhat similar to all the other simulation runs is the amount of energy spent for the transmission power setting. At lower transmission power more energy is spent, as transmission power is increased (from $6 \mathrm{~dB}$ to $10 \mathrm{~dB}$ ) less energy is spent. This is not attributed to failed transmissions and the need for retransmissions because the UDP protocol was used at the transport layer. UDP was specifically chosen as the transport layer protocol so that retransmissions would not influence the data (because retransmissions do not occur). Figure 13 depicts the results.

Table 15 presents a summary of the number of sent packets from node 0 to node 14 . Each row represents a simulation run using a different transmission power setting. Also shown in Table 15 is the total energy remaining at both the sender and the receiver when the simulations completed.

\begin{tabular}{ccccc}
\hline \multicolumn{5}{c}{ Node 0 transmitting to Node 14 } \\
\hline \multicolumn{4}{c}{ Packets } & \multicolumn{1}{c}{ Energy Remaining } \\
\hline $\boldsymbol{T}_{\boldsymbol{x} \text { Power }}$ & Sent & Received & Sender & Receiver \\
\hline-3 & 494 & 92 & 593.157 & 593.337 \\
-2 & 494 & 70 & 593.223 & 593.427 \\
-1 & 494 & 130 & 592.965 & 593.305 \\
0 & 494 & 201 & 592.992 & 593.419 \\
1 & 494 & 198 & 592.676 & 593.453 \\
2 & 494 & 261 & 592.559 & 593.393 \\
3 & 494 & 190 & 592.395 & 593.491 \\
4 & 494 & 255 & 592.345 & 593.679 \\
5 & 494 & 285 & 592.548 & 593.723 \\
6 & 494 & 427 & 607.081 & 608.503 \\
7 & 494 & 436 & 660.285 & 661.885 \\
8 & 494 & 451 & 709.893 & 711.417 \\
9 & 494 & 470 & 754.832 & 756.307 \\
10 & 494 & 478 & 795.164 & 796.192 \\
\hline
\end{tabular}

Table 15. Summary of the results under Scenario $2 b$.

\begin{tabular}{ccccc}
\hline \multicolumn{5}{c}{ Node 3 transmitting to Node 17 } \\
\hline \multicolumn{4}{c}{ Packets } & \multicolumn{1}{c}{ Energy Remaining } \\
\hline $\boldsymbol{T}_{\boldsymbol{x} \text { Power }}$ & Sent & Received & Sender & Receiver \\
\hline-3 & 494 & 124 & 593.154 & 593.350 \\
-2 & 494 & 124 & 593.156 & 593.420 \\
-1 & 494 & 167 & 593.040 & 593.310 \\
0 & 494 & 189 & 593.075 & 593.434 \\
1 & 494 & 204 & 593.069 & 593.461 \\
2 & 494 & 229 & 593.018 & 593.548 \\
3 & 494 & 255 & 592.901 & 593.588 \\
4 & 494 & 283 & 592.712 & 593.675 \\
5 & 494 & 390 & 592.237 & 593.693 \\
6 & 494 & 396 & 606.756 & 608.463 \\
7 & 494 & 449 & 660.329 & 661.894 \\
8 & 494 & 477 & 710.049 & 711.414 \\
9 & 494 & 491 & 755.104 & 756.279 \\
10 & 494 & 494 & 795.081 & 796.195 \\
\hline
\end{tabular}

Table 16. Summary of the results under Scenario $2 b$.
Table 16 presents a summary of sent packets from node 3 to node 17 , like the previous table each row represents a simulation run using a different transmission power setting.

\subsection{Scenario $2 \mathrm{c}$}

The final variation of Scenario 2 was to implement dynamic transmission power (i.e. Scenario $2 c$ ). In Scenario $2 a$ and $2 b$ the simulations ran for 500 seconds on a fixed transmission power, thus with 14 transmission power settings, 14 simulations were run each for 500 seconds. In this scenario the interest was to determine how energy depletion and performance would be impacted if transmission power was changing whist the simulation was running or 'on the fly'. Therefore, Scenario $1 c$ consisted of a single simulation with varying transmission power. The transmission power was increased every $24 \mathrm{~s}$ and begins at $-10 \mathrm{~dB}$ incrementing to $+10 \mathrm{~dB}$. Table 17 reminds the reader of the scenario settings with the addition information regarding the duration of the increase time of transmission power.

\begin{tabular}{ll}
\hline \multicolumn{2}{c}{ Scenario 1c } \\
\hline Placement Model & $6 \times 3$ Grid \\
Distance between Nodes & 100 Meters \\
Mobility Model & Constant \\
Transport Protocol & UDP \\
Data Rate & $2048 \mathrm{~B} / \mathrm{s}$ \\
Packet Size & $2048 \mathrm{Bytes}$ \\
Transmission Power & $-10 \mathrm{~dB}$ to $+10 \mathrm{~dB}$ \\
Transmission Power Increment & 24 seconds \\
Data Capture Interval & 5 seconds \\
Simulation Duration & 504 seconds \\
\hline
\end{tabular}

Table 17. Setting under Scenario 2c.

Table 18 presents a summary of the data every 24 seconds and Figure 14 shows the number of packets sent and received with the energy spent at the sender and the receiver for each transmission power setting for the communication between node 0 (sender) and node 14 (receiver). Table 19 and Figure 15 show the same summary information for communication between node 3 (sender) and node 17 (receiver).

\begin{tabular}{|c|c|c|c|c|}
\hline \multicolumn{5}{|c|}{ Node 0 \& Node 14} \\
\hline & \multicolumn{2}{|c|}{ Packets } & \multicolumn{2}{|c|}{ Energy Spent } \\
\hline$T_{x \text { Power }}$ & Sent & Received & Sender & Receiver \\
\hline-10 & 23 & 0 & 19.657 & 19.658 \\
\hline-9 & 24 & 0 & 19.656 & 19.656 \\
\hline-8 & 24 & 0 & 19.657 & 19.656 \\
\hline-7 & 24 & 0 & 19.656 & 19.657 \\
\hline-6 & 24 & 0 & 19.656 & 19.656 \\
\hline-5 & 24 & 0 & 19.657 & 19.658 \\
\hline-4 & 24 & 0 & 19.695 & 19.694 \\
\hline-3 & 24 & 0 & 19.737 & 19.725 \\
\hline-2 & 24 & 4 & 19.779 & 19.764 \\
\hline-1 & 24 & 0 & 19.756 & 19.740 \\
\hline 0 & 24 & 0 & 19.788 & 19.744 \\
\hline 1 & 24 & 24 & 19.743 & 19.727 \\
\hline 2 & 24 & 13 & 19.718 & 19.701 \\
\hline
\end{tabular}




\begin{tabular}{ccccc}
\hline 3 & 24 & 0 & 19.848 & 19.733 \\
4 & 24 & 16 & 19.780 & 19.697 \\
5 & 24 & 1 & 19.972 & 19.721 \\
6 & 24 & 0 & 20.058 & 19.722 \\
7 & 24 & 24 & 19.774 & 19.687 \\
8 & 24 & 24 & 19.779 & 19.711 \\
9 & 24 & 24 & 19.791 & 19.702 \\
10 & 24 & 24 & 19.846 & 19.729 \\
\hline
\end{tabular}

Table 18. Results under Scenario 2c.

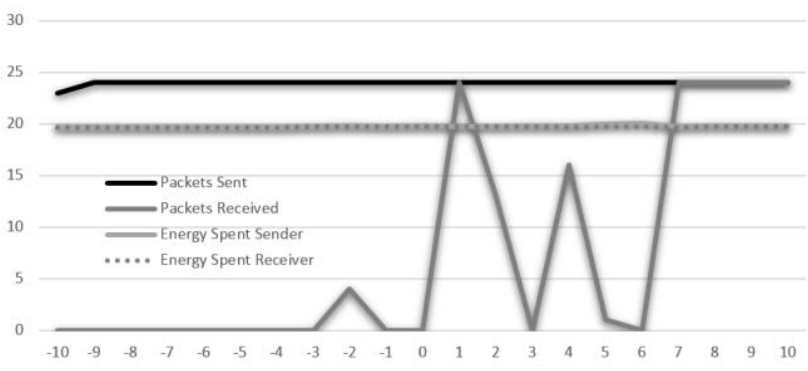

Figure 14. Remaining Energy vs Transmission Power under Scenario 2c for Nodes 0 and Node 14.

Whilst transmission power was between $-10 \mathrm{~dB}$ and -3 $\mathrm{dB}$ no packets were received by either destination (tables 18 and 19 and figures 14 and 15). Then at $-2 \mathrm{~dB}$ some packets were received but $83 \%$ approx. were lost, this is true for both destinations. At $0 \mathrm{~dB}$ no packets were received which was also true of both destinations. At $1 \mathrm{~dB}$ the number of received packet for both destinations significantly improves and transmissions are heard by the destinations, node 14 receives $100 \%$ of sent packets and node 17 receives $83 \%$ of packets. At $2 \mathrm{~dB}$ the number of received packets decreases for both destinations, node 14 receives $54.2 \%$ and node 17 receives $45.8 \%$. At $3 \mathrm{~dB}$ there is a contrast between the number of packets received at each destination with Node 14 receiving no packets and Node 17 receiving $79.2 \%$. Then at $4 \mathrm{~dB}$ the reverse happens, Node 14 receives $66.6 \%$ and Node 17 receives $41.6 \%$. At $5 \mathrm{~dB}$ and $6 \mathrm{~dB}$ no packets are received for either destination (except Node 14 does receive 1 packet at $5 \mathrm{~dB}$ ). Node 14 receives all packets that have been sent when transmission power is set between $7 \mathrm{~dB}$ and $10 \mathrm{~dB}$. Node 17 does not receive any packets at $7 \mathrm{~dB}$ and around $20.8 \%$ at $8 \mathrm{db}$. At $9 \mathrm{~dB}$ and $10 \mathrm{~dB}$ all packets are received at Node 17.

\begin{tabular}{|c|c|c|c|c|}
\hline \multicolumn{5}{|c|}{ Node 3 \& Node 17} \\
\hline & \multicolumn{2}{|c|}{ Packets } & \multicolumn{2}{|c|}{ Energy Spent } \\
\hline$T_{x \text { Power }}$ & Sent & Received & Sender & Receiver \\
\hline-10 & 23 & 0 & 19.658 & 19.657 \\
\hline-9 & 24 & 0 & 19.656 & 19.657 \\
\hline-8 & 24 & 0 & 19.656 & 19.656 \\
\hline-7 & 24 & 0 & 19.657 & 19.656 \\
\hline-6 & 24 & 0 & 19.657 & 19.657 \\
\hline-5 & 24 & 0 & 19.657 & 19.657 \\
\hline-4 & 24 & 0 & 19.694 & 19.660 \\
\hline
\end{tabular}

\begin{tabular}{ccccc}
\hline-3 & 24 & 0 & 19.734 & 19.684 \\
-2 & 24 & 4 & 19.783 & 19.724 \\
-1 & 24 & 0 & 19.757 & 19.724 \\
0 & 24 & 0 & 19.791 & 19.715 \\
1 & 24 & 22 & 19.745 & 19.727 \\
2 & 24 & 11 & 19.708 & 19.701 \\
3 & 24 & 19 & 19.777 & 19.736 \\
4 & 24 & 10 & 19.712 & 19.696 \\
5 & 24 & 0 & 19.721 & 19.720 \\
6 & 24 & 0 & 19.719 & 19.721 \\
7 & 24 & 0 & 19.680 & 19.679 \\
8 & 24 & 5 & 19.717 & 19.708 \\
9 & 24 & 24 & 19.794 & 19.700 \\
10 & 24 & 24 & 19.839 & 19.734 \\
\hline
\end{tabular}

Table 19. Results under Scenario $2 c$ (Node 3 and Node 17).

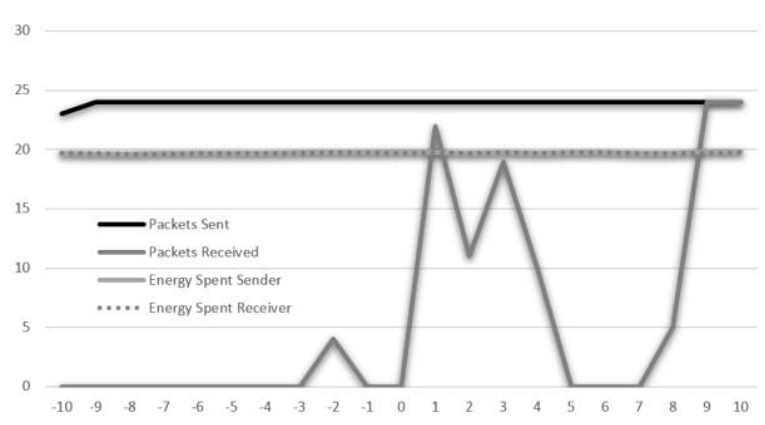

Figure 15. Remaining Energy vs Transmission Power under Scenario 2c for Nodes 3 and Node 17.

\section{Discussion}

The original hypothesis was simple; reduce transmission power to the maximal optimal (the maximum transmission power required for successful stable communication) and produce a greener energy efficient network that can operate over a longer lifetime. Calculations show that with nodes spaced around $100 \mathrm{~m}$ apart the best transmission power would be $-2 \mathrm{~dB}$ which gave a maximum optimal of 108 meters, a maximum of 110 meters and a mean of 107 meters (Table 20).

\begin{tabular}{cccc}
\hline \multicolumn{3}{c}{ Distance } \\
\hline $\boldsymbol{T}_{\boldsymbol{x} \text { Power }}$ & $\begin{array}{c}\text { Max } \\
\text { Optimal }\end{array}$ & Max & Min \\
\hline-20 & 14 & 14 & 12 \\
-19 & 16 & 16 & 14 \\
-18 & 18 & 18 & 16 \\
-17 & 20 & 20 & 18 \\
-16 & 22 & 22 & 20 \\
-15 & 25 & 25 & 23 \\
-14 & 26 & 28 & 25 \\
-13 & 32 & 32 & 30 \\
-12 & 35 & 35 & 33 \\
-11 & 40 & 40 & 38 \\
-10 & 44 & 44 & 42 \\
-9 & 48 & 50 & 47 \\
-8 & 52 & 55 & 52 \\
-7 & 60 & 64 & 59 \\
-6 & 66 & 71 & 66 \\
-5 & 74 & 82 & 75 \\
\hline
\end{tabular}




\begin{tabular}{|c|c|c|c|}
\hline-4 & 83 & 91 & 85 \\
\hline-3 & 95 & 99 & 96 \\
\hline-2 & 108 & 110 & 107 \\
\hline-1 & 122 & 124 & 121 \\
\hline 0 & 133 & 138 & 134 \\
\hline 1 & 148 & 167 & 151 \\
\hline 2 & 167 & 182 & 168 \\
\hline 3 & 185 & 200 & 191 \\
\hline 4 & 210 & 223 & 215 \\
\hline 5 & 232 & 247 & 241 \\
\hline 6 & 260 & 283 & 270 \\
\hline 7 & 295 & 310 & 304 \\
\hline 8 & 339 & 347 & 342 \\
\hline 9 & 367 & 400 & 382 \\
\hline 10 & 410 & 460 & 430 \\
\hline 11 & 461 & 506 & 481 \\
\hline 12 & 525 & 564 & 539 \\
\hline 13 & 585 & 623 & 605 \\
\hline 14 & 657 & 729 & 678 \\
\hline 15 & 736 & 796 & 763 \\
\hline 16 & 829 & 930 & 864 \\
\hline 17 & 908 & 1020 & 967 \\
\hline 18 & 1025 & 1161 & 1084 \\
\hline 19 & 1148 & 1280 & 1215 \\
\hline 20 & 1286 & 1417 & 1355 \\
\hline
\end{tabular}

Table 20. Transmission Power vs Distance.

However, from the analysis of the data produced by the simulations it is evident that although nodes are in communication range at $-2 \mathrm{~dB}$ performance is somewhat poor. Table 21 presents an overview of the performance for each of the simulation runs at $-2 \mathrm{~dB}$.

\begin{tabular}{ccccc}
\hline & \multicolumn{2}{c}{ Packets } & \multicolumn{2}{c}{ Energy Remaining } \\
\hline Scenario & Sent & Received & Sender & Receiver \\
\hline $1 \mathrm{a}$ & 494 & 103 & 594.592 & 594.592 \\
$1 \mathrm{~b}$ & 494 & 351 & 594.592 & 594.592 \\
$1 \mathrm{c}$ & 24 & 4 & 594.590 & 594.590 \\
$2 \mathrm{a}(0-14)$ & 494 & 57 & 592.697 & 592.860 \\
$2 \mathrm{a}(3-17)$ & 494 & 172 & 592.586 & 593.317 \\
$2 \mathrm{~b}(0-14)$ & 494 & 70 & 593.223 & 593.427 \\
$2 \mathrm{~b}(3-17)$ & 494 & 124 & 593.156 & 593.420 \\
$2 \mathrm{c}(0-14)$ & 24 & 4 & 19.779 & 19.764 \\
$2 \mathrm{c}(3-17)$ & 24 & 4 & 19.783 & 19.724 \\
\hline
\end{tabular}

Table 21. Packets and Remaining Energy at $-2 \mathrm{~dB}$.

At $-3 \mathrm{~dB}$ results were as expected, in most cases no packets were received with the exception for Scenarios $1 \mathrm{~b}$ and $2 \mathrm{~b}$. According to the transmission range vs distance table a transmission power set to $-3 \mathrm{~dB}$ could achieve at best 99 meters, with a maximal optimal at 95 meters and a mean of 96 meters. Some successful communication at -3 $\mathrm{dB}$ for Scenarios $1 \mathrm{~b}$ and $2 \mathrm{~b}$ is not so unexpected though because the random waypoint mobility model had been applied. Thus, the nodes begin at 100 meters apart but would have moved within the 95 - $99 \mathrm{~m}$ limits and at those periods successful communication could occur.

The most reliable communication was at much higher transmission levels, see Table 22, which summaries the lowest transmission power for the highest number of received packets.

\begin{tabular}{cccccc}
\hline & \multicolumn{2}{c}{ Packets } & \multicolumn{2}{c}{ Energy Remaining } \\
\hline Scenario & $\boldsymbol{T}_{\boldsymbol{x} \text { Power }}$ & Sent & Received & Sender & Receiver \\
\hline 1a & $8 \mathrm{~dB}$ & 494 & 493 & 710.640 & 711.660 \\
1b & 6dB & 494 & 351 & 608.175 & 609.013 \\
1c & $8 \mathrm{~dB}$ & 24 & 24 & 19.757 & 19.687 \\
2a $(0-14)$ & $8 \mathrm{~dB}$ & 494 & 494 & 710.387 & 711.421 \\
2a $(3-17)$ & $8 \mathrm{~dB}$ & 494 & 494 & 710.398 & 711.398 \\
$2 \mathrm{~b}(0-14)$ & $10 \mathrm{~dB}$ & 494 & 478 & 795.164 & 796.192 \\
$2 \mathrm{~b}(3-17)$ & $10 \mathrm{~dB}$ & 494 & 494 & 795.081 & 796.195 \\
$2 \mathrm{c}(0-14)$ & $7 \mathrm{~dB}$ & 24 & 24 & 19.774 & 19.687 \\
$2 \mathrm{c}(3-17)$ & $9 \mathrm{~dB}$ & 24 & 24 & 19.794 & 19.700 \\
\hline
\end{tabular}

Table 22. Highest Number of Packets Received and Lowest Transmission Power.

The nature of the experiment involved communication within a MANET rather than mobile nodes in communication with an access point. This meant the routing of packets through intermediate nodes along a path to a destination occurs. One could assume that increasing the transmission power increased the power of the signal (thus the distance that signal could propagate), and as such, required fewer intermediate nodes for packets to traverse to the destination. This assumption would be correct.

However, in Scenario 1 the world consisted of a $200 \mathrm{~m}$ x $200 \mathrm{~m}$ geographical area. Therefore, for node 0 to be in direct communication range of node 8 (each at opposite ends of the world) we can calculate their distance using the Pythagorean theorem.

$$
\sqrt{\left(x_{2}-x_{1}\right)^{2}+\left(y_{2}-y_{1}\right)^{2}}
$$

This yields a distance between the source node and destination node of $\approx 282.84$ meters. According to the transmission vs. distance table, a transmission power of 7 $\mathrm{dB}$ could easily accommodates this distance (i.e. a maximum optimal of 295, with a maximum of 310 and a mean of 304). However, Scenarios $1 a$ and $1 c$ required a transmission distance of at least $8 \mathrm{~dB}$ for optimal performance. Scenario $1 b$ only required $6 \mathrm{~dB}$ but as stated earlier the nodes would have moved within closer proximity.

In Scenario 2 the world consisted of a $500 \mathrm{~m} \times 200 \mathrm{~m}$ geographical area, however, node 0 transmitted to node 14 and node 3 transmitted to node 17, which gave the distance between the two communicating parties as $\approx 282.84 \mathrm{~m}$, so would have been easily in range of their respective destinations at $7 \mathrm{~dB}$. However, for the most optimal packet delivery ratio at least $8 \mathrm{~dB}$ was required for Scenario $2 a$. Scenario $2 c$ showed optimal performance with a transmission setting of $7 \mathrm{~dB}$ and $9 \mathrm{~dB}$ respectively for each transmitting party. Scenario $2 b$ with the random waypoint mobility model applied required a transmission power setting of $10 \mathrm{~dB}$. 
These results show that ideally nodes should be in direct communication range for optimal performance with a transmission power setting higher than the maximum optimal. The reason for this is because in a MANET, packets are routed through intermediate nodes and as a result, routing algorithms generate their own routing traffic to discover and maintain routes, in addition to this routing information is propagated throughout the network. The higher than required transmission power setting helps with this noisy communication medium.

The main goal of this research was to show the most optimal transmission power to conserve energy in a MANET to make that network 'greener' and to increase the lifetime of that network.

Thus, we are interested in the energy remaining at the end of each simulation run. Table 23 presents a table that shows the transmission power setting that conserves the most energy (energy began at 1000).

\begin{tabular}{cccccc}
\hline & \multicolumn{2}{c}{ Packets } & \multicolumn{2}{c}{ Energy Remaining } \\
\hline Scenario & $\boldsymbol{T}_{\boldsymbol{x} \text { Power }}$ & Sent & Received & Sender & Receiver \\
\hline $1 a$ & $10 \mathrm{~dB}$ & 494 & 493 & 795.185 & 796.342 \\
$1 b$ & $10 \mathrm{~dB}$ & 494 & 493 & 795.159 & 796.331 \\
$1 c$ & $10 \mathrm{~dB}$ & 24 & 24 & 19.811 & 19.698 \\
$2 a(0-14)$ & $10 \mathrm{~dB}$ & 494 & 494 & 794.963 & 796.172 \\
$2 a(3-17)$ & $10 \mathrm{~dB}$ & 494 & 494 & 794.986 & 796.143 \\
$2 b(0-14)$ & $10 \mathrm{~dB}$ & 494 & 478 & 795.164 & 796.192 \\
$2 b(3-17)$ & $10 \mathrm{~dB}$ & 494 & 494 & 795.081 & 796.195 \\
$2 c(0-14)$ & $10 \mathrm{~dB}$ & 24 & 24 & 19.846 & 19.729 \\
$2 c(3-17)$ & $10 \mathrm{~dB}$ & 24 & 24 & 19.839 & 19.734 \\
\hline
\end{tabular}

Table 23. Packets and Remaining Energy at $-2 \mathrm{~dB}$.

Table 23 shows that increasing the transmission power conserves energy. The likely reason for this is because traffic propagates over greater distances so there is less call on the route discovery process, thus conserving energy by propagating less routing information.

\section{Conclusion}

In this research, it has been shown that there is an optimal transmission power setting that results in both greater network performance regarding packet delivery ratio and less energy consumption. Therefore, the network performs optimally whilst conserving energy, which results in a greener more energy efficient network. The research consisted of an analysis of 72 simulations (both with static transmission power and dynamically changing transmission power) and from this analysis we conclude that all simulations resulted in a consistent message.

Future research could involve moving away from setting uniformly transmission power output to determine if an optimal transmission power setting can be obtained my making better use of intermediate nodes. This would account for nodes that do not need to transmit signals as far as other nodes. It would also be interesting to research the power consumptions across different protocols, for example, does the Temporally Ordered Routing Algorithm (TORA) routing protocol use as much energy as the Destination-Sequenced Distance Vector (DSDV) routing protocol in establishing the routes? This research builds upon many others' research, for example, [11-12, 14, 1718 ] but with the added feature of being able to determine an optimal transmitting power in a MANET.

\section{Acknowledgements}

We thank all staff of the School of Computer Science, University of Manchester, the Department of Computing Science and Mathematics, University of Stirling, the Institut National Des Sciences Appliquees and the Department of Mathematics \& Computer Science, Liverpool Hope University.

\section{References}

[1] J.B. Hughes, P. Lazaridis, I. Glover and A. Ball, "A survey of link quality properties related to transmission power control protocols in wireless sensor networks," $23^{\text {rd }}$ International Conference on Automation and Computing (ICAC), Huddersfield, 1-5, 2017.

[2] J.B. Hughes, P. Lazaridis, I. Glover and A. Ball, "Opportunities for transmission power control protocols in wireless sensor networks," $23^{\text {rd }}$ International Conference on Automation and Computing (ICAC), Huddersfield, 1-5, 2017.

[3] W.R. Heinzelman, A. Chandrakasan and H. Balakrishnan, "Energy-efficent communication protocol for wireless microsensor networks," in Proc. Hawaii Int. Conf. System Sciences, Maui, Hawaii, 2000.

[4] W. He, S. Nabar, and R. Poovendran. "An energy framework for the network simulator 3 (ns-3)." In Proc. $4^{\text {th }}$ International Conference on Simulation Tools and Techniques (ICST), 222-230, 2011.

[5] D. Halperin, B. Greenstein, A. Sheth, and D. Wetherall. Demystifying $802.11 \mathrm{n}$ power consumption. In Proc. International Conference on Power Aware Computing and Systems (HotPower'10), Berkeley, CA, USA, 2010.

[6] A. Zarei Ghanavati and D. Lee, "Optimizing the Bit Transmission Power for Link Layer Energy Efficiency under Imperfect CSI," in IEEE Transactions on Wireless Communications, 99, 1-1.

[7] R. Kotian, G. Exarchakos and A. Liotta, "Reliable lowpower wireless networks over unstable transmission power," IEEE $14^{\text {th }}$ International Conference on Networking, Sensing and Control (ICNSC), Calabria, 801-806, 2017.

[8] P.P. Priyesh and S.K. Bharti, "Dynamic transmission power control in wireless sensor networks using P-I-D feedback control technique," $9^{\text {th }}$ International Conference on Communication Systems and Networks (COMSNETS), Bengaluru, 306-313, 2017.

[9] U. Ghosh and R. Datta, "A Secure Addressing Scheme for Large-Scale Managed MANETs," in IEEE Transactions on Network and Service Management, 12(3), 483-495, Sept. 2015.

[10] E. Gromova, D. Gromov, N. Timonin, A. Kirpichnikova and S. Blakeway, "A Dynamic Game of Mobile Agent Placement in a MANET," International Conference on 
Systems Informatics, Modelling and Simulation (SIMS), Riga, 153-158, 2016.

[11] Tiwari and I. Kaur, "Performance evaluation of energy efficient for MANET using AODV routing protocol," $3^{\text {rd }}$ International Conference on Computational Intelligence \& Communication Technology (CICT), Ghaziabad, 1-5, 2017.

[12] M. Ilango and A.V.S. Kumar, "Deterministic multicast linkbased energy optimized routing in MANET," $2^{\text {nd }}$ International Conference on Electrical, Computer and Communication Technologies (ICECCT), Coimbatore, Tamil Nadu, India, 1-10, 2017.

[13] W. Feng, H. Luo, B. Sun and C. Gui, "Performance analysis of sliding window network coding for energy efficient in MANETs," $7^{\text {th }}$ IEEE International Conference on Electronics Information and Emergency Communication (ICEIEC), Macau, 219-222, 2017.

[14] S. Prajapati, N. Patel and R. Patel, "Optimizing Performance of OLSR Protocol Using Energy Based MPR Selection in MANET," $5^{\text {th }}$ International Conference on Communication Systems and Network Technologies, Gwalior, 268-272, 2015.

[15] W. Feng, H. Luo, B. Sun and C. Gui, "Performance analysis of sliding window network coding for energy efficient in MANETs," $7^{\text {th }}$ IEEE International Conference on Electronics Information and Emergency Communication (ICEIEC), Macau, 2017, 219-222.

[16] H.T. Friis, "A Note on a Simple Transmission Formula," in Proc. of IRE, 34(5), 254-256, May 1946.

[17] M. Lanati, D. Curone, E.L. Secco, G. Magenes, P. Gamba, An autonomous long range monitoring system for emergency operators, International Journal of Wireless \& Mobile Networks, 3(1), 10-23, 2011.

[18] E.L. Secco, D. Curone, A. Tognetti, A. Bonfiglio, G. Magenes, Validation of Smart Garments for Physiological and Activity-Related Monitoring of Humans in Harsh Environment, American Journal of Biomedical Engineering, 2(4), 189-196, 2012. 